\title{
Diabetes and Aboriginal vision health Le diabète et la santé oculaire des Autochtones
}

\author{
BY / PAR A. PAUL CHRIS, OD, VISION INSTITUTE OF CANADA
}

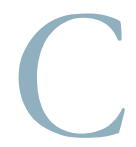

anada is home to an Aboriginal population of of whom $61 \%$ are First Nations, $34 \%$ are Métis (mixed nativeEuropean descent), and 5\% are Inuit. These three distinct groups all have unique "local geographic and linguistic heritages, cultural practices and spiritual beliefs." Slightly more than half live in urban areas but maintain strong connections to their communities of origin. ${ }^{1}$ Although there has been an increase in the number of Aboriginal people living in urban areas, there has actually been a net migration back to First Nations communities in the last 40 years. $^{2}$ There are 615 native communities (reserves or bands) in Canada. British Columbia has the largest number of reserves at 198 followed by Ontario with 153 . Ontario has more remote First Nations communities than any other region.

Aboriginal people make up $3.8 \%$ of the Canadian population, ranking second in the world to New Zealand, where the Maori people make up 15\% of the population. In the United States and Australia, approximately $2 \%$ of the general population is Aboriginal. ${ }^{2}$ According to the 2006 Aboriginal Census, Ontario has the largest native population $(243,000)$, followed by BC $(196,000)$, Alberta $(188,000)$, Manitoba $(175,000)$, Saskatchewan $(142,000)$, and Quebec $(108,000)$. The remaining 25,000 live in the other provinces and territories. ${ }^{3}$

The Aboriginal community is also the largest growing segment of the Canadian population, increasing at a rate six times faster than non-Aboriginal people. Almost half the native population is below 25 years of age, compared to 40 years for the non-native population.

Aboriginal history in Canada reflects years of government forced assimilation and colonization efforts with the "appropriation of land and loss of traditional livelihoods." The residential school system, established in 1892, resulted in the mandatory removal of children from their homes and their placement in boarding schools where they were "forbidden to speak their own languages." Many suffered emotional, physical, and sexual abuse, turning to drugs and alcohol in later life to deal with their trauma. The attendant loss of self-esteem and the destruction of family bonds and parenting skills have caused a cultural shock resulting in ill health, poverty and family breakdown. ${ }^{4}$
One of the most significant consequences of the "psychosocial stress" associated with colonization and the loss of traditional foods and lifestyles is the epidemic of diabetes that is eroding the health and lives of Aboriginal people. The extent to which Aboriginal people have been affected is both complex and astonishing. This is a phenomenon affecting indigenous people worldwide. The longterm complications associated with diabetes, such as blindness, heart disease, kidney disease, infectious disease and amputations, are an emerging public health crisis. $^{5}$

Before 1950, diabetes was rare in native communities. ${ }^{6}$ Diabetes was not detected in 1500 First Nations people who underwent a tuberculosis survey in Saskatchewan in 1937. ${ }^{7}$ Today twenty percent of the Canadian Aboriginal population lives with diabetes, a number that has doubled in the last two decades, most likely due to environmental (nutrition and lifestyle) factors. ${ }^{6,7}$ Across Canada, type 2 diabetes is three to five times higher in Aboriginal people than in the general population. According to a Saskatchewan study published in January 2010, the rate of diabetes among 


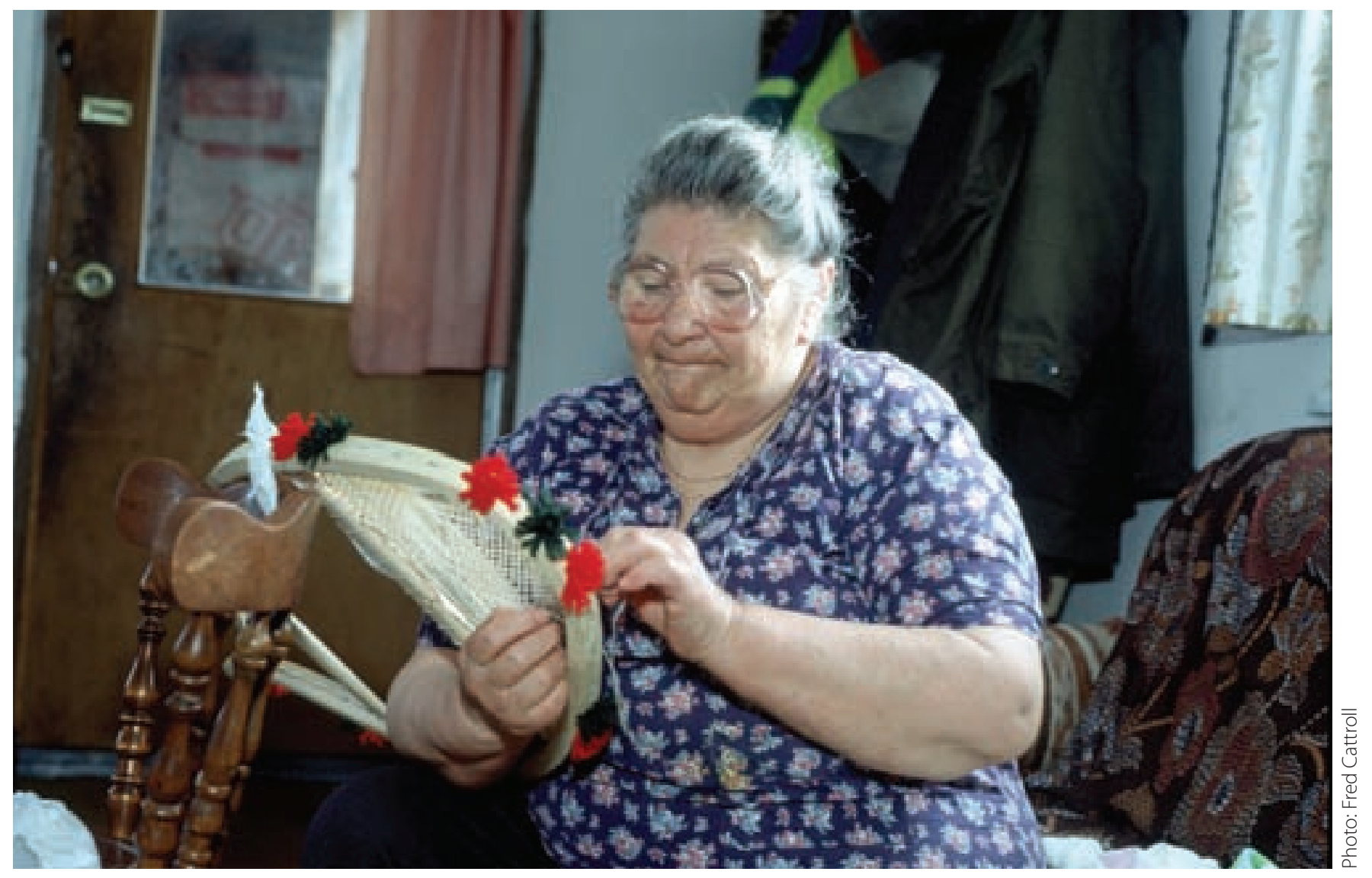

Aboriginal women of childbearing age is four times greater than women in the general population. Native women also have much higher rates of gestational diabetes, which dramatically increases a woman's risk of developing diabetes later in life, and also makes her offspring more prone to the disease.

Diabetes is the leading cause of adult blindness in Canada. According to one report, the rate of progression and severity of diabetic retinopathy, unlike kidney disease, is no greater in Aboriginal people than that of the general population. ${ }^{8}$ As recently as 2005 , there was limited data on the prevalence of diabetic retinopathy in Aboriginal Canadians. A study published that year involving the Sandy Lake First Nations community in Northern Ontario reported the following prevalence rates: nonproliferative diabetic retinopathy 24\% (NPDR), macular edema $(5 \%)$ and proliferative diabetic retinopathy $2 \%$ (PDR). ${ }^{9}$

These findings were consistent with an earlier study in 2002 by Maberley, et al. ${ }^{10}$ The authors of the 2005 study suggested that the relatively low prevalence rates of macular edema and PDR "possibly reflect low median duration of diabetes or the presence of protective genetic factors."
A more recent report from 2007, the Southern Alberta Study of Diabetic Retinopathy, showed that prevalence rates of diabetic retinopathy in type 2 diabetes in native and non-native subjects were identical, with a prevalence rate of $40 \%$, "far higher" than the Sandy Lake study. Native subjects also tended to have more advanced retinopathy changes indicating that Aboriginal ethnicity does play a role in the severity of retinal complications. ${ }^{11}$

Despite conflicting research, what is clear is that with the disproportionate and increasing number of native people with diabetes, and its occurrence at a much early age than the general 
- 74 percent of people who have diabetes for 10 years or more will develop some form of diabetic retinopathy.

- Approximately 14 percent of people with diabetes have diabetic macular edema and prevalence increases to 29 percent for people with diabetes who use insulin for more than 20 years.

- Left untreated, 25 percent of people with diabetic macular edema will develop moderate vision loss within three years.

- Estimates of the rate of annual eye exams vary greatly by country and study, but the rate of screening is generally fairly low (from 40 to 65 percent).

- Worldwide guidelines (for people with diabetes) recommend annual screenings with a dilated eye exam from an eye care specialist. ${ }^{12}$

- International Diabetes Federation

population, diabetes will lead to a more significant burden of preventable vision loss in Aboriginal communities than in non-Aboriginal groups.

There are few statistics available to show the rate of annual dilated eye examinations being received by Aboriginal Canadians. If trends from other services are any indication, access to annual dilated eye exams for many Aboriginal people with diabetes is limited by geography and the availability of an optometrist or ophthalmologist. Several telemedicine projects using digital retinal cameras have been established but are not filling the need for the diagnostic vision care services that is required. There is also a lack of published data on the relationships between diabetic retinopathy, macular edema severity and visual acuity. ${ }^{13}$

In the United States the National Eye Institute was created by Congress in 1968 as part of the National Institutes of Health. In 1991 it established the National Eye Health Education Program which released a report in 2004 titled: American Indian and Alaska Native Diabetic Eye Disease Communication Plan. This communication plan was designed to improve the eye health of American Indians and Alaska Natives with diabetes and to raise awareness about the importance of annual dilated eye exams in this population. ${ }^{14}$

An environmental scan produced in 2007 by the National Collaborating Centre on Aboriginal Health based at the University of Northern British Columbia, states:

"...a review of the international literature suggests that Canada is well behind other countries in addressing Aboriginal eye health and vision care services. Both the United States and Australia have developed innovative, Aboriginal specific, community-controlled programs and promotional material..." 15

Canada has a larger native population, by percentage, than the United States but is indeed well behind in addressing the Aboriginal vision health issues that are becoming an emerging public health crisis. More Canadian funding and research are required to fill the gap in scientific knowledge about
Aboriginal vision health.

Optometry, its partners and professional organizations, need to work with Aboriginal people and their organizations to create an effective Canadian communication strategy to help educate Aboriginal health care workers and eye care professionals about Aboriginal eye health issues and the importance of annual dilated eye exams for native Canadians living with diabetes.

1 Macaulay AC. Improving aboriginal health: How can health care professional contribute? Can Fam Phys. Vol. 55: April 2009

2 Bailey S; Native population growing. The Canadian Press; Jan 15, 2008

3 Atkinson DL Preschool Vision Screening and Aboriginal Eye Health: An Environmental Scan and Literature Review. BC Initiatives; April 2007

4 Macaulay AC. Improving aboriginal health: How can health care professional contribute? Can Fam Phys. Vol 55: April 2009

5 Hanley AJ. Diabetes in Indigenous Peoples: Medscape Diabetes and Endocrinology. July 2006; http:/ / cme. medscape.com/viewarticle/540921

6 Young TK, et al. Type 2 diabetes mellitus in Canada's First Nations: status of an epidemic in progress. CMAJ; September 5, 2000; 163 (5)

7 Dyck R, et al. Epidemiology of diabetes mellitus among First Nations and nonFirst Nations adults. CMAJ February 23, 2010 182(3)

8 Harris SB. Diabetes in indigenous peoples: Program and abstracts of the American Diabetes Association 66th Scientific Sessions; June 9-13, 2006; Washington, DC.

9 Hanley AJG, et al. Complications of Type 2 Diabetes among Aboriginal Canadians Prevalence and associated risk 
factors. Diabetes Care; August $2005 \mathrm{Vol}$ 28 no. 8 2054-2057

10 Maberley D, et al. Digital photographic screening for diabetic retinopathy in the James Bay Cree. Ophthalmic Epidemiol 9: 169-178, 2002

11 Ross SA, et al. Diabetic Retinopathy in Native and Nonnative Canadians. Exp Diab Res. Vol 2007: Article ID 76271

12 International Diabetes Federation. http://www.idf.org/internationaldiabetes-federation website accessed July 15, 2010.

13 Tucker D, et al. Investigation the links between diabetic retinopathy, macular edema severity and visual acuity in patients with diabetes. Expert Review of Ophthalmology; Dec 2008 Vol 3 No 6 (673-688)

14 National Eye Health Education Program: American Indian and Alaska Native Diabetic Eye Disease Communication Plan. US Department of Health and Human Services: National Eye Institute; January 2004

15 Atkinson DL. Preschool Vision Screening and Aboriginal Eye Health: An Environmental Scan and Literature Review. BC Initiatives; April 2007

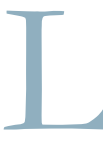

e Canada compte une population autochtone qui dépasse 1,2 million de personnes parmi lesquelles $61 \%$ sont des membres des Premières nations, $34 \%$ des Métis (personnes de descendance mixte autochtoneeuropéenne) et $5 \%$ des Inuits. Ces trois groupes distincts présentent tous des « héritages linguistiques et géographiques régionaux, des pratiques culturelles et des croyances spirituelles » qui leur sont propres. Un peu plus de la moitié d'entre eux vivent dans des régions urbaines et entretiennent des liens étroits avec leur communauté d'origine(1). Bien que le nombre d'Autochtones vivant en région urbaine s'accroisse, les dernières 40 années ont été marquées par un solde migratoire positif en faveur des communautés des Premières nations. ${ }^{2}$ Le Canada compte 615 communautés autochtones (réserves ou bandes). La Colombie-Britannique est la province qui compte le plus grand nombre de réserves (198) devant l'Ontario (153). L'Ontario a davantage de communautés de Premières nations isolées que toutes les autres régions.

Les Autochtones représentent $3,8 \%$ de la population canadienne, un chiffre qui les classe au second rang mondial devant la Nouvelle-Zélande où le peuple Maori représente $15 \%$ de la population. Aux ÉtatsUnis et en Australie, environ $2 \%$ de la population générale est autochtone. ${ }^{2}$ Selon le recensement sur les Autochtones de 2006, l'Ontario est la province qui abrite la plus grande population autochtone (243 000), suivie par la Colombie-Britannique (196 000), l’Alberta (188 000), le
Manitoba (175 000), la Saskatchewan (142 000) et le Québec (108 000). Le restant de cette population (25 000) dans d'autres provinces et territoires. ${ }^{3}$

La collectivité autochtone est également le segment de la population canadienne ayant la plus forte croissance, avec un taux six fois plus rapide que celui des personnes non autochtones. Près de la moitié de la population autochtone a moins de 25 ans, un chiffre à confronter aux 40 ans de la population non autochtone.

L'histoire des Autochtones au Canada est liée à des années d'assimilation forcée et à des entreprises de colonisation menées par le gouvernement qui ont conduit à « l'appropriation de leurs territoires et la perte de leurs moyens de subsistance ». Le système de pensionnat, mis en place en 1892, s'est traduit par des mesures contraignantes comme l'enlèvement des enfants de leur foyer et leur placement dans des pensionnats où il leur était « interdit de parler leur propre langue ». Nombre d'entre eux ont souffert de violences psychologique, physique et sexuelle et se sont tournés plus tard dans leur vie vers la consommation de drogues ou d'alcool pour pouvoir surmonter ces traumatismes. Les pertes d'estime de soi, la destruction des liens familiaux et la détérioration des 
compétences parentales que tout cela suppose ont provoqué un choc culturel, à l'origine de problèmes de santé, de la pauvreté et de l'éclatement familial. ${ }^{4}$

L'une des conséquences marquantes de ce «stress psychosocial », qui s'ajoute aux effets de la colonisation et à la perte des nourritures traditionnelles et des modes de vie, est l'épidémie de diabète qui mine la santé et la vie des Autochtones. Il est stupéfiant de constater à quel point les Autochtones ont été touchés par cette épidémie dont les causes sont complexes. Il s'agit d'un phénomène qui affecte tous les peuples autochtones dans le monde. Les complications à long terme associées au diabète, comme la cécité, les cardiopathies, les néphropathies, les maladies infectieuses et les amputations, constituent une situation de crise naissante en termes de santé publique. $^{5}$

Avant 1950, le diabète restait une affection rare dans les communautés autochtones. ${ }^{6}$ Le diabète n'a d'ailleurs pas été détecté parmi les 1500 membres des Premières nations qui firent l'objet d'un suivi de la tuberculose en Saskatchewan en 1937.7 Aujourd'hui, c'est $20 \%$ de la population autochtone canadienne qui vit avec le diabète, un pourcentage qui a doublé au cours des deux dernières décennies, vraisemblablement en raison de facteurs environnementaux (la nutrition et le style de vie)., Partout au Canada, on constate que le taux de diabète de type 2 est de trois à cinq fois plus élevé chez les Autochtones que dans la population générale. Selon une étude réalisée en Saskatchewan et publiée en janvier 2010, le taux de diabète chez les femmes autochtones en âge de procréer est quatre fois supérieur à celui des femmes de la population générale. Les femmes autochtones présentent également des taux de diabète gestationnel bien supérieurs, lesquels augmentent de façon spectaculaire le risque pour une femme de voir se développer un diabète plus tard au cours de sa vie, mais aussi de rendre sa descendance plus encline à cette maladie. ${ }^{7}$

Le diabète est la cause principale de la cécité chez l'adulte au Canada. Selon une étude, la vitesse de progression et la sévérité de la rétinopathie diabétique, contrairement aux néphropathies, ne serait pas plus grande parmi les Autochtones que dans la population générale. ${ }^{8}$ Pas plus tard qu'en 2005, on ne disposait que de peu de données sur la prévalence de la rétinopathie diabétique chez les Autochtones du Canada. Une étude publiée cette année sur la communauté de Premières nations Sandy Lake, située dans le Nord de l'Ontario, a révélé les taux de prévalence suivants : $24 \%$ pour la rétinopathie diabétique non proliférante (RDNP), $5 \%$ pour l'œdème maculaire et $2 \%$ pour la rétinopathie diabétique proliférante (RDP). ${ }^{9}$

Ces résultats correspondent à ceux d'une étude antérieure menée en 2002 par Maberley et coll. ${ }^{10}$ Les auteurs de l'étude de 2005 ont avancé l'hypothèse que les taux de prévalence relativement bas en matière d'œdème maculaire et de
RDP « pouvaient refléter de courtes durées moyennes de diabète ou la présence de facteurs de protection génétique $»{ }^{9}$

Un rapport plus récent datant de 2007 (Southern Alberta Study of Diabetic Retinopathy) a montré que le taux de prévalence de la rétinopathie diabétique chez les sujets autochtones et non autochtones atteints d'un diabète de type 2 était équivalent dans ces deux populations, soit $40 \%$, un taux « bien supérieur » à celui révélé par l'étude de la communauté Sandy Lake. Les sujets autochtones sont également plus enclins à présenter des évolutions de rétinopathie plus rapides, ce qui montrerait que leur origine ethnique joue un rôle dans la gravité des complications rétiniennes. ${ }^{11}$

En dépit de résultats de recherche contradictoires, il est désormais clair que l'on fait face à un nombre croissant et disproportionné de personnes autochtones atteintes du diabète et, d'autre part, que cette maladie survient à un stade bien plus précoce que dans la population générale; on sait en outre que le diabète va entraîner davantage de cas évitables de perte de vision, au sein des communautés autochtones que dans les groupes non autochtones.

Il existe peu de statistiques qui rendent compte du taux annuel d'examen de la vision à pupille dilatée dont bénéficient les Autochtones canadiens. Si les tendances qui émanent d'autres services sont de quelques enseignements, force est de constater que l'accès de nombreux Autochtones atteints de diabète aux examens annuels de la 
- $74 \%$ des personnes atteintes de diabète depuis au moins 10 ans vont développer une forme quelconque de rétinopathie diabétique.

- Environ $14 \%$ des personnes atteintes de diabète présentent des œdèmes maculaires d'origine diabétique; cette prévalence s'accroît à $29 \%$ pour les personnes atteintes de diabète qui utilisent l'insuline depuis plus de 20 ans.

- Sans traitement, $25 \%$ des personnes qui souffrent d'un œdème maculaire d'origine diabétique développeront une perte de vision modérée d'ici à trois ans.

- Les estimations qui ont été réalisées sur les taux annuels d'examen de la vision varient de manière importante selon les pays et les études, mais le taux de dépistage est généralement assez bas (de 40 à $65 \%$ ).

- Les lignes directrices mondiales en la matière recommandent de procéder à des dépistages annuels en faisant passer aux personnes atteintes de diabète un examen de la vision à pupille dilatée, conduit par un spécialiste des soins oculovisuels. ${ }^{12}$

- Fédération internationale du diabète

vision à pupille dilatée est limité par la situation géographique et la disponibilité d'un optométriste ou d'un ophtalmologiste. Plusieurs projets de télémédecine s'appuyant sur l'utilisation de caméras rétiniennes numériques ont été mis en place, mais ils ne suffisent pas encore à combler le besoin en services de diagnostic et de soins de la vue. Peu de données ont en outre été publiées sur les liens entre la rétinopathie diabétique, la gravité des œdèmes maculaires et l'acuité visuelle. ${ }^{13}$ Aux États-Unis, le Congrès a créé en 1968 le National Eye Institute comme entité du National Institutes of Health. En 1991, cet institut a mis en place le National Eye Health Education Program qui a publié en 2004 un rapport intitulé : American Indian and Alaska Native Diabetic Eye Disease Communication Plan. Ce plan de communication a été élaboré pour améliorer la santé oculaire des Indiens d'Amérique et des Autochtones de l'Alaska atteints par le diabète et pour sensibiliser davantage ces populations à l'importance de subir un examen annuel de la vision à pupille dilatée. ${ }^{14}$

Voilà ce qu'affirme une analyse de la conjoncture réalisée en 2007 par le Centre de collaboration de la santé autochtone abrité par l'University of Northern British Columbia :

«...une analyse de la littérature internationale conduit à penser que le Canada se situe loin derrière d'autres pays en ce qui concerne la prestation de services de santé oculaire et de soins de la vue aux Autochtones. Les ÉtatsUnis et l'Australie ont tous deux élaborés des programmes novateurs, dédiés aux Autochtones et gérés par les communautés; ils ont également conçus des documents de promotion... $»^{15}$

Bien que le Canada compte une population autochtone plus grande qu'aux États-Unis, il se situe bien derrière son voisin dans le règlement des problèmes de santé oculaire des Autochtones qui sont en voie de deve- nir de nouveaux problèmes de santé publique. Le Canada doit mobiliser davantage de fonds et consentir plus d'efforts de recherche pour combler son écart en matière de connaissance scientifique sur la santé oculaire des Autochtones. Le secteur de l'optométrie, ses partenaires et les organisations professionnelles doivent travailler de concert avec les Autochtones et leurs organisations pour développer une stratégie de communication canadienne plus efficace. Il s'agit d'aider à sensibiliser les travailleurs autochtones en soins de santé, ainsi que les professionnels des soins oculo-visuels aux problèmes de santé des Autochtones et à l'importance de faire passer un examen annuel de la vision à pupille dilatée aux Canadiens autochtones souffrant du diabète.

1 Macaulay AC. Améliorer la santé des Autochtones : Quelle contribution les professionnels de la santé peuvent-ils apporter? Le médecin de famille canadien. Vol. 55 : avril 2009

2 Bailey S; Native population growing. La Presse Canadienne; Jan 15, 2008

3 Atkinson DL Preschool Vision Screening and Aboriginal Eye Health : An Aboriginal Eye Health and Literature Review. BC Initiatives; avril 2007

4 Macaulay AC. Améliorer la santé des Autochtones : Quelle contribution les professionnels de la santé peuvent-ils apporter? Le médecin de famille canadien. Vol. 55 : avril 2009

5 Hanley AJ. Diabetes in Indigenous Peoples : Medscape Diabetes and Endocrinology. Juillet 2006; http:// cme.medscape.com/viewarticle/540921 
${ }^{\text {mAlrex }}$

(loteprednol etabonate ophthalmic suspension $0.2 \% \mathrm{w} / \mathrm{v}$ )

\section{Prescribing Summary}

\section{Patient Selection Criteria}

\section{THERAPEUTIC CLASSIFICATION}

Corticosteroid

\section{INDICATIONS AND CLINICAL USE}

Alrex ${ }^{\circledast}$ (loteprednol etabonate) 0phthalmic Suspension is indicated for temporary short-term relief of the signs and symptoms of seasonal allergic conjunctivitis CONTRAINDICATIONS

Suspected or confirmed infection of the eye: viral diseases of the cornea and conjunctiva including epithelial herpes simplex keratitis (dendritic keratitis), vaccinia, and varicella; untreated ocular infection of the eye; mycobacterial infection of the eye and fungal diseases of ocular structures; hypersensitivity to this drug or any ingredient in the formulation or container, or to other corticosteroids.

\section{SPECIAL POPULATIONS}

\section{Use in Pediatrics ( $<18$ years of age):}

Alrex ${ }^{\circledast}$ should not be used in pediatric patients.

Use in Geriatrics:

Alrex ${ }^{\circledast}$ should not be used in geriatric patients. The safety and efficacy of Alrex ${ }^{\circledast}$

have not been established in patients $>65$ years of age.

Pregnant Women:

Alrex ${ }^{\circledast}$ should not be used in pregnant women, unless the benefit clearly outweighs the risks. Studies in pregnant women have not been conducted.

Nursing Women:

Alrex ${ }^{\circledast}$ should not be used in lactating women, unless the benefit clearly outweighs the risks.

\section{Safety Information}

\section{WARNINGS AND PRECAUTIONS}

\section{General}

For ophthalmic, short-term use only (up to 14 days).

The initial prescription and renewal of Alrex ${ }^{\circledast}$ should be made by a physician only after appropriate ophthalmologic examination is performed. If signs and symptoms fail to improve after two days, the patient should be re-evaluated. If Alrex ${ }^{\circledast}$ is used for 10 days or longer, intraocular pressure should be closely monitored.

Prolonged use of corticosteroids may result in cataract and/or glaucoma formation. Alrex ${ }^{\circledast}$ should not be used in the presence of glaucoma or elevated intraocular pressure, unless absolutely necessary and close ophthalmologic monitoring is undertaken. Extreme caution should be exercised, and duration of treatment should be kept as short as possible.

Alrex ${ }^{\circledast}$ should not be used in cases of existing (suspected or confirmed) ocular viral, fungal, or mycobacterial infections. Alrex ${ }^{\oplus}$ may suppress the host response and thus increase the hazard of secondary ocular infections. The use of Alrex ${ }^{\circledast}$ in patients with a history of herpes simplex requires great caution and close monitoring. Alrex ${ }^{\circledast}$ contains benzalkonium chloride.

Alrex ${ }^{\circledast}$ has not been studied in pregnant or nursing women, but has been found to be teratogenic in animals. Alrex ${ }^{\circledast}$ should not be used in pregnant or nursing women unless the benefits clearly outweigh the risks.

\section{Carcinogenesis and Mutagenesis}

Long-term animal studies have not been conducted to evaluate the carcinogenic potential of loteprednol etabonate. Loteprednol etabonate was not genotoxic in vitro in the Ames test, the mouse lymphoma tk assay, or in a chromosome aberration test in human lymphocytes, or in vivo in the single dose mouse micronucleus assay. Ophthalmologic

Alrex ${ }^{\circledR}$ should be used as a brief temporary treatment. If Alrex ${ }^{\circledast}$ is used for 10 days or longer, intraocular pressure should be closely monitored. The initial prescription and renewal of Alrex ${ }^{\circledast}$ should be made by a physician only after appropriate ophthalmologic examination is performed, ie. slit lamp biomicroscopy or fluorescein staining if appropriate. If signs and symptoms fail to improve after two days, the patient should be re-evaluated.

Prolonged use of corticosteroids may result in glaucoma with damage to the optic nerve, defects in visual acuity and fields of vision, and in posterior subcapsular cataract formation. Alrex ${ }^{\circledast}$ should not be used in the presence of glaucoma or elevated intraocular pressure, unless absolutely necessary and careful and close appropriate ophthalmologic monitoring (including intraocular pressure and lens clarity) is undertaken.

Corneal fungal infections are particularly prone to develop coincidentally with long-term local steroid application. Fungus invasion must be considered in any persistent corneal ulceration involving steroid use. Fungal cultures should be taken when appropriate.

Prolonged use of corticosteroids may suppress the host response and thus increase the hazard of secondary ocular infections. In those diseases causing thinning of the cornea or sclera, perforations have been known to occur with the use of topical steroids. In acute purulent conditions of the eye, steroids may mask infection or enhance existing infection.

Use of ocular steroids may prolong the course and may exacerbate the severity of many viral infections of the eye (including herpes simplex). Employment of a corticosteroid medication in the treatment of patients with a history of herpes simplex requires great caution.

Formulations with benzalkonium chloride should be used with caution in soft contact lens wearers.

\section{ADVERSE REACTIONS}

Overview

Reactions associated with ophthalmic steroids include elevated intraocular pressure, which may be associated with optic nerve damage, visual acuity and field defects, posterior subcapsular cataract formation, secondary ocular infection from pathogens including herpes simplex, and perforation of the globe where there is thinning of the cornea or sclera.

In nineteen clinical trials ranging from 1 to 42 days in length, 1,209 patients received various concentrations of loteprednol etabonate in topical ocular drops $(0.005 \%, 0.05 \%, 0.1 \%, 0.2 \%, 0.5 \%)$. Adverse events related to loteprednol etabonate were generally mild to moderate, non-serious and did not interrupt continuation in the studies. The most frequent ocular event reported as related to therapy was increased IOP: 6\% (77/1209) in patients receiving loteprednol etabonate, as compared to $3 \%(25 / 806)$ in the placebo treated patients. With the exception of elevations in IOP, the incidence of events in the LE group was similar to, or less than that of the placebo control groups. Itching was reported as related to therapy in 3\% of the loteprednol treated eyes, injection, epiphora, burning/stinging other than at instillation, foreign body sensation, and burning/stinging at instillation were each reported for $2 \%$ of eyes. The most frequent non-ocular event reported as related to therapy was headache, reported for $1.2 \%$ of the loteprednol treated subjects and $0.6 \%$ of the placebo treated subjects.

To report an adverse event, contact your Regional Adverse Reaction Monitoring office at 1-866-234-2345 or Bausch \& Lomb at 1-888-459-5000

\section{$\triangle \theta$ Administration}

One drop instilled into the affected eye(s) four times daily for up to 14 days. If scheduled dose is missed, patient should be advised to wait until the next dose and then continue as before.

SHAKE VIGOROUSLY BEFORE USING. Alrex ${ }^{\circledast}$ should be stored upright between $15^{\circ}-25^{\circ} \mathrm{C}$ for up to 28 days after first opening.

The preservative in Alrex ${ }^{\circledast}$, benzalkonium chloride, may be absorbed by soft contact lenses, and can discolour soft contact lenses. Therefore, Alrex ${ }^{\circledR}$ should not be used while the patient is wearing soft contact lenses. Patients who wear soft contact lenses and whose eyes are not red should wait ten to fifteen minutes after instilling Alrex ${ }^{\circledast}$ before they insert their contact lenses.

Patients should be advised not to wear a contact lens if their eye is red. Alrex ${ }^{\circledast}$ should not be used to treat contact lens related irritation.

\section{SUPPLEMENTAL PRODUCT INFORMATION}

\section{WARNINGS AND PRECAUTIONS}

Sexual Function/Reproduction

The effects of Alrex $x^{\mathscr{E}}$ on sexual function and reproduction have not been studied in humans. Treatment of male and female rats with up to $50 \mathrm{mg} / \mathrm{kg} /$ day and $25 \mathrm{mg} / \mathrm{kg} /$ day of loteprednol etabonate, respectively, (1000 and 500 times the Alrex ${ }^{\infty}$ clinical dose) prior to and during mating, was clearly harmful to the rats, but did not impair their copulation 
performance and fertility (i.e., ability of female rats to become pregnant). However, these doses were highly toxic and had significant toxic effects on the pregnancies, and the survival and development of the offspring. Maternal toxicity, possible occurrence of abnormalities and growth retardation started at 10 times the Alrex ${ }^{\mathscr{D}}$ clinical dose.

Neurologic

Disturbances and suppression of the Hypothalamic-Pituitary-Adrenal (HPA) axis can occur with systemic exposure to corticosteroids. However, given the very low systemic exposure to loteprednol etabonate when using Alrex as directed, these possible effects are not likely.

\section{Endocrine and Metabolism}

Glucocorticoids, mostly when systemic exposure occurs, decrease the hypoglycemic activity of insulin and oral hypoglycemics, so that a change in dose of the antidiabetic drugs may be necessitated. In high doses, glucocorticoids also decrease the response to somatotropin. The usual doses of mineralocorticoids and large doses of some glucocorticoids cause hypokalemia and may exaggerate the hypokalemic effects of thiazides and high-ceiling diuretics. In combination with amphotericin-B, they also may cause hypokalemia. Glucocorticoids appear to enhance the ulcerogenic effects of non-steroidal anti-inflammatory drugs. They decrease the plasma levels of salicylates, and salicylism may occur on discontinuing steroids. Glucocorticoids may increase or decrease the effects of prothrombopenic anticoagulants. Estrogens, phenobarbital, phenytoin and rifampin increase the metabolic clearance of adrenal steroids and hence necessitate dose adjustments.

However, given the very low systemic exposure to loteprednol etabonate when using Alrex ${ }^{\oplus}$ as directed, these possible effects are not likely.

Immune

Cortisol and the synthetic analogs of cortisol have the capacity to prevent or suppress the development of the local heat, redness, swelling, and tenderness by which inflammation is recognized. At the microscopic level, they inhibit not only the early phenomena of the inflammatory process (edema, fibrin deposition, capillary dilation, migration of leukocytes into the inflamed area, and phagocytic activity) but also the later manifestations, such as capillary proliferation fibroblast proliferation, deposition of collagen, and, still later, cicatrisation. Clinical Trial Adverse Drug Reactions Possibly or probably related adverse events from two Phase III studies are listed below:

\begin{tabular}{|c|c|c|}
\hline & $\begin{array}{c}\text { Alrex }^{\mathbb{E}} 0.2 \% \\
\mathrm{~N}=133\end{array}$ & $\begin{array}{l}\text { Placebo } \\
\mathrm{N}=135\end{array}$ \\
\hline \multicolumn{3}{|l|}{ SPECIAL SENSES (EYE DISORDERS) } \\
\hline \multicolumn{3}{|l|}{ Intraocular Pressure } \\
\hline - elevation of 6 to $9 \mathrm{~mm} \mathrm{Hg}$ & $2 \%$ to $12 \%$ & $0 \%$ to $6 \% *$ \\
\hline - elevation of $\geq 10 \mathrm{~mm} \mathrm{Hg}$ & $1(1 \%)$ & $1(1 \%)$ \\
\hline Chemosis & $6(5 \%)$ & $7(5 \%)$ \\
\hline Vision, Abnormal or Blurred & $4(3 \%)$ & $5(4 \%)$ \\
\hline Burning/stinging, on instillation & $3(2 \%)$ & $6(4 \%)$ \\
\hline Itching Eye & $3(2 \%)$ & $3(2 \%)$ \\
\hline Dry Eye & $2(2 \%)$ & $4(3 \%)$ \\
\hline Burning/stinging, not on instillation & $2(2 \%)$ & $2(1 \%)$ \\
\hline Epiphora & $1(1 \%)$ & $9(7 \%)$ \\
\hline Discharge & $1(1 \%)$ & $3(2 \%)$ \\
\hline Foreign Body Sensation & $1(1 \%)$ & $1(1 \%)$ \\
\hline Discomfort Eye & $1(1 \%)$ & $0(0 \%)$ \\
\hline Injection & $1(1 \%)$ & $0(0 \%)$ \\
\hline Eye Pain & $1(1 \%)$ & $0(0 \%)$ \\
\hline Sticky Eye & $0(0 \%)$ & $7(5 \%)$ \\
\hline Erythema Eyelids & $0(0 \%)$ & $2(1 \%)$ \\
\hline Eye Disorder & $0(0 \%)$ & $2(1 \%)$ \\
\hline \multicolumn{3}{|l|}{ BODY AS A WHOLE } \\
\hline Face Edema (Head) & $1(1 \%)$ & $0(0 \%)$ \\
\hline Allergic Reaction & $1(1 \%)$ & $0(0 \%)$ \\
\hline \multicolumn{3}{|l|}{ MUSCULOSKELETAL SYSTEM } \\
\hline Twitching & $0(0 \%)$ & $1(1 \%)$ \\
\hline
\end{tabular}

One patient in the Alrex group and one patient in the placebo group experienced increases in $10 \mathrm{P}$ of $\geq 10 \mathrm{~mm} \mathrm{Hg}$. Among these, one in each group had an IOP increase of $\geq 15 \mathrm{~mm} \mathrm{Hg}$, reaching IOP values over $30 \mathrm{~mm} \mathrm{Hg}$. In both studies, there were more patients with IOP increases of 6 to $9 \mathrm{~mm} \mathrm{Hg}$ in the Alrex group than in the placebo group (see table below). In study A, among the patients with IOP increases of 6 to $9 \mathrm{~mm} \mathrm{Hg}$, four reached an IOP value of 22 to $23 \mathrm{~mm} \mathrm{Hg}$, and one patient reached $29 \mathrm{~mm} \mathrm{Hg}$ and was discontinued (clinically significant increase in IOP). All these five patients were from the Alrex ${ }^{\mathbb{1}}$ groups.

\section{Incidence of IOP increases of 6 to $9 \mathrm{~mm} \mathrm{Hg}$ from baseline} (number of patients and percentages)

\begin{tabular}{|cccc|}
\hline & Day 7 & $\begin{array}{c}\text { Duration of treatment } \\
\text { Day 14 }\end{array}$ & Day 28 \\
\hline $\begin{array}{c}\text { Alrex } \\
\text { Study-A } \\
\text { Study-B }\end{array}$ & $\begin{array}{l}6(9 \%) \\
3(5 \%)\end{array}$ & $\begin{array}{l}6(9 \%) \\
1(2 \%)\end{array}$ & $\begin{array}{c}8(12 \%) \\
4(6 \%)\end{array}$ \\
\hline $\begin{array}{c}\text { Placebo } \\
\text { Study-A }\end{array}$ & $0(0 \%)$ & $4(6 \%)$ & $1(2 \%)$ \\
Study-B & $0(\%)$ & $\begin{array}{c}(6 \%) \\
0(\%)\end{array}$ & $0(\%)$ \\
\hline
\end{tabular}

Due to the sample size for each arm of the two phase III studies in SAC, all events captured are greater than $1 \%$ of $n$.

\section{SYMPTOMS AND TREATMENT OF OVERDOSAGE}

For management of suspected accidental oral ingestion or drug overdose, consult your regional poison control centre. No cases of overdose have been reported.

Full Product Monograph available for health professionals at: http://www.bausch.ca

\section{BAUSCH + LOMB}

๑) 2008 Bausch \& Lomb Canada Incorporated. Vaughan Ontario L4K 4B4

rm/® Denote trademarks of Bausch \& Lomb Incorporated or its affiliates.

6 Young TK, et coll. Type 2 diabetes mellitus in Canada's First Nations : status of an epidemic in progress. JAMC; 5 septembre 2000; 163 (5)

7 Dyck R, et coll. Epidemiology of diabetes mellitus among First Nations and non-First Nations adults. JAMC, 23 février 2010 182(3)

8 Harris SB. Diabetes in indigenous peoples : Program and abstracts of the American Diabetes Association 66th Scientific Sessions; 9-13 juin 2006; Washington, DC.

9 Hanley AJG, et coll. Complications of Type 2 Diabetes among Aboriginal Canadians : Prevalence and associated risk factors. Diabetes Care; août 2005 Vol 28 no 8 2054-2057

10 Maberley D, et coll. Digital photographic screening for diabetic retinopathy in the James Bay Cree. Ophthalmic Epidemiol 9 : 169-178, 2002

11 Ross SA, et coll. Diabetic Retinopathy in Native and Nonnative Canadians. Exp Diab Res. Vol 2007 : Article ID 76271

12 Fédération internationale du diabète. Consultation en ligne sur le site Web : http://www.idf.org/internationaldiabetes-federation, 15 juillet 2010.

13 Tucker D, et coll. Investigating the links between diabetic retinopathy, macular edema severity and visual acuity in patients with diabetes. Expert Review of Ophthalmology; décembre 2008, vol. 3, no 6 (673-688)

14 National Eye Health Education Program : American Indian and Alaska Native Diabetic Eye Disease Communication Plan. US Department of Health and Human Services : National Eye Institute; janvier 2004

15 Atkinson DL. Preschool Vision Screening and Aboriginal Eye Health : An Environmental Scan and Literature Review. BC Initiatives; avril 2007 